\title{
Digitalisierungsstrategien in Privatarchiven: Herausforderungen und Chancen
}

\author{
Elke Huwiler
}

\author{
Gerade Archivare sollten zur Kenntnis nehmen, dass ihr alter Leitspruch \\ "Quod non est in actis, non est in mundo " \\ einen markanten Bedeutungswandel erlebt hat: \\ "Quod non est in rete, non est in mundo». \\ (Gillner 2013, S. 408)
}

\section{Einleitung}

Im 21. Jahrhundert kann kein Archiv es sich leisten, Fragen der Digitalisierung ausser Acht zu lassen. 2015 formulierte die Zeitschrift Archivar die Entwicklungsschritte im Zusammenhang mit der " Digitalisierung des Archivguts » noch folgendermassen: «Schien es anfangs noch zu genügen, allgemeine Informationen wie Adresse und Öffnungszeiten eines Archivs online zu stellen, so forderten die Nutzerinteressen bald schon die Bereitstellung von Beständeübersichten und elektronischen Findmitteln im Netz. Inzwischen sind auch Archivalien in digitaler Form hinzugetreten, was eine weltweite Nutzungsperspektive für das einzelne Archiv eröffnete. " ${ }^{1}$ Als weiterer Entwicklungsschritt ist nun die Möglichkeit der Verknüpfung digitaler Metadaten und Inhalte zwischen verschiedenen Institutionen durch den Bereich der Linked Open Data hinzugekommen. Es geht heute nicht mehr nur darum, reaktiv den geänderten Ansprüchen der NutzerInnen Rechnung tragen, sondern auch (pro)aktiv die Chance zu nutzen, die eine Digitalisierung für das Archiv bietet.

Wenn ein Archiv sich dazu entschliesst, umfassend zu digitalisieren, müssen die Ziele klar definiert und die Möglichkeit der Umsetzung des Vorhabens geprüft werden - es braucht idealerweise eine Strategie. Der folgende Beitrag beschreibt die wichtigsten Aspekte einer solchen Digitalisierungsstrategie für Archive ${ }^{2}$ -

\footnotetext{
Pilger 2015, S. 213.

Der Beitrag befasst sich primär mit der digitalen Aufbereitung analogen Sammelguts im Archiv. Dabei handelt es sich um eine retrospektive Digitalisierung von bereits in Handschrift oder Druck (oder Tonband oder Film) vorhandenem Material. Dieser Vorgang wird auch Retrodigitalisierung genannt (Heckmann 2006, S. 123). Das Produkt eines solchen Vorgangs ist ein Digitalisat, d.h. ein digitales Abbild eines analog vorhandenen Objekts.

Im vorliegenden Beitrag ist mit dem Begriff «Digitalisat » somit immer das durch das Archiv hergestellte Digitalisat eines analogen Objekts gemeint, und „Digitalisieren“ bezeichnet in diesem Zusammenhang auch primär das digitale Aufbereiten solchen Materials.
} 
insbesondere Archive mit privaten Nachlässen - und zeigt diesbezügliche Herausforderungen und Chancen auf. ${ }^{3}$

\section{Aspekte einer Digitalisierungsstrategie}

Prozesse von Archivgutdigitalisierung können sehr unterschiedlich aussehen, je nach Digitalisierungsstrategie, die gewählt wird. Diese Wahl wiederum hängt von unterschiedlichen Kriterien ab. Allgemein lassen sich laut Berger alle «Entscheidungen, die einer Digitalisierung zugrunde liegen [...], auf vier Fragen reduzieren: Kann das Archivale digitalisiert werden? [...] Soll das Archivale, der Bestand oder der Teilbestand digitalisiert werden? [...] Wie soll digitalisiert werden? [...] In welcher Reihenfolge soll digitalisiert werden? » Die Beantwortung dieser Fragen auf einer strategischen Ebene ist ein erster wichtiger Schritt für ein Archiv, wenn es « klare nicht von Einzelstücken abhängige Regeln [schaffen] und feste Strukturen " vorgeben will. ${ }^{4}$

Andererseits geht durch die Vorgabe solcher festen Strukturen auch ein gewisser Verlust an Flexibilität einher. Wie starr solchen Regeln und Strukturen bei der Digitalisierung gefolgt werden soll und ob diese nicht von Fall zu Fall wieder modifiziert werden können, ist eine weitere Entscheidung, die in eine Digitalisierungsstrategie Eingang finden kann. ${ }^{5}$ Beim SLA und DLA wurden hier beispielsweise zwei gänzlich unterschiedliche Wege gewählt: Während das SLA seinen Digitalisierungsprozess jeweils an das zu digitalisierende Korpus anpasst und von Fall zu Fall vorgeht, hat das DLA zunächst ein Digitalisierungszentrum geschaffen und will die Strategie möglichst zentralisieren.

Zentrale Aspekte, die in die Formulierung einer Digitalisierungsstrategie einfliessen müssen, sind das Selbstverständnis als Institution sowie Vision und Ziele der Digitalisierung. Eine weitsichtig ausgerichtete Vision und eine Besinnung auf das eigentliche Selbstverständnis der Institution können helfen, die Digitalisierung nicht als Hürde, sondern als Chance zu sehen, ganz im Sinne eines ,Mindshifts' $:$ «When it comes to digitising the archival holdings it is often expressed that it is an impossible task to digitise each document. This may be true, but we know very little about the future

3 Der Beitrag stützt sich auf meine Masterarbeit zu den Digitalisierungsstrategien des Schweizerischen Literaturarchivs in Bern (SLA) und des Deutsche Literaturarchiv in Marbach (DLA). Da mir für diese Masterarbeit vom SLA und vom DLA internes, nicht zu veröffentlichendes Material zur Verfügung gestellt wurde, verschiebt sich der Fokus des vorliegenden Beitrags auf eine allgemeine Beschreibung von Aspekten, die eine entsprechende Digitalisierungsstrategie berücksichtigen muss. Es werden nur punktuell die spezifischen entsprechenden Strategien des SLA und des DLA beschrieben.

5 So hat sich beispielsweise das Historische Archiv der Stadt Köln dagegen entscheiden, einem deduktiven Vorgehen zu folgen, das eine vorgängig festgelegte Strategie verlangt hätte. Vielmehr wollte man zuerst „aus der praktischen Arbeit heraus eigene Erfahrungen sammeln“. Es wurde in einem induktiven Verfahren aufgrund einer „Behandlung einer überschaubaren Zahl von Einzelstücken [...] eine Auswahlmatrix" erzeugt, die dann auf die weiteren Archivalien angewendet wurde. Berger 2015, S. 25. 
technical and logistical solutions, and in a world where information must be digital to be relevant, we need a mind shift. If you instead say that the goal is to digitise everything, without taking into account whether it is possible or not, you start looking for new approaches and solutions. " ${ }^{6}$ Digitalisiertes Archivgut und die Möglichkeit für Nutzende, dieses online zu konsultieren, dürften für die Ausstrahlung eines Archivs nach aussen durchwegs als positiv bewertet werden: « the creation of a digital archival collection and access to it can promote the image of the archival institution, demonstrating at the same time the importance of the collection and increasing awareness of the public and other organizations. ${ }^{7}$ In einer Ausformulierung einer Vision können Gedächtnisinstitutionen sich diesbezüglich positionieren.

Bei der Formulierung der Ziele ist zu unterscheiden zwischen auf Fernziele hin formulierte Digitalisierungspläne eines Archivs und der Formulierung einer Strategie, die in der Regel eine konkrete Umsetzbarkeit im Zeithorizont von wenigen Jahren vorsieht. In der Praxis werden diese Aspekte jedoch nicht immer unterschieden, weil mögliche Einschränkungen bereits bekannt sind und in die Zielformulierung mit einbezogen werden. Solche möglichen Einschränkungen werden vor allem von den Ressourcen (Finanzen, Personal und Infrastruktur) geleitet. Mattmann nennt die " nicht geringen Kosten einer retrospektiven Digitalisierung » den meistgenannten Einwand gegen diese. ${ }^{8}$ Ein weiterer Aspekt, der ebenfalls oft zu Einschränkungen bei der Formulierung von Zielen einer Digitalisierung führt, sind die juristische Grundlagen. Eine flächendeckende Digitalisierung und digitalisierte Bereitstellung ist für (private) Archive unter anderem aus juristischen Gründen ungleich schwieriger als für Bibliotheken. ${ }^{9}$ Bei der konkreten Durchführung von Digitalisierungsprojekten im Archiv ist eine genaue Prüfung der juristischen Implikationen unerlässlich: Persönlichkeitsrecht, Urheberrecht und anderweitig gemachte vertragliche Absprachen in Bezug auf das zu digitalisierende Material müssen abgeklärt werden.

Digitalisate herzustellen zum Schutz der Originale und zum besseren Zugriff der Archive auf die Materialien zwecks deren Bereitstellung für eine beschränkte Nutzung ist juristisch jedoch jederzeit möglich. Dabei wird der Zugriff derart gestaltet, dass eine individuelle Identifizierung der Benutzenden nötig ist und die Materialien nicht weiterverbreitet werden können. In diesem Fall « entspricht die Beeinträchtigung des Betroffenen weitgehend dem Versand von Archivalien oder Reproduktionen in gedruckter Form, welcher im geltenden Archivrecht ausdrücklich vorgesehen ist, weshalb diese Art der Zugangseröffnung zu retrospektiv digitalisierten Archivalien über das Internet vom geltenden Archivrecht gedeckt wäre. »10 So ist es auch laut

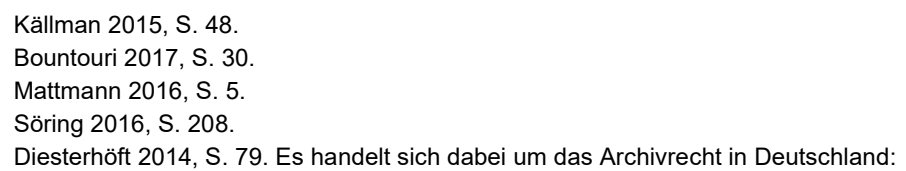


dem neu geregelten, erweiterten Archivprivileg der Schweiz unter dem Sicherheitsund Erhaltungsaspekt im Prinzip möglich, Bestände zu digitalisieren, die aus juristischen Gründen nicht frei zur Verfügung gestellt werden können: das « Archivprivileg (Art. 24 Abs. 1bis URG) » ermächtigt dazu, « die zur Sicherung und Erhaltung ihrer Bestände notwendigen Werkexemplare herzustellen, und zwar sowohl in analoger als auch in digitaler Form. »11 Es ist somit nicht so, dass juristische Gründe ein Privatarchiv davon abhalten würden, auch urheberrechtlich geschützte Bestände zu digitalisieren. In der Praxis geschieht dies jedoch nicht oder nur sehr selten, und wenn, dann unter konservatorischem Aspekt. Die Schweizer Nationalbibliothek, der das SLA unterstellt ist, hält in ihren Leitlinien für das SLA ausdrücklich fest, dass nur die « Sammlungen oder Teile aus den Beständen des SLA [...], deren Rechtsstatus eine Aufschaltung zulässt », digitalisiert werden dürfen. ${ }^{12}$ So war die Digitalisierung der Rilke-Korrespondenz durch das SLA nur möglich, weil « die Urheberrechte an den Werken und der Korrespondenz Rilkes 70 Jahre nach dessen Tod am 29. Dezember 1926 - also bereits seit 1997 - abgelaufen sind. » Und aus « denselben Gründen wurden alle Zeugnisse, die nicht von Rilkes Hand stammen, für dieses Projekt generell nicht berücksichtigt. ॥ 13

In einer Digitalisierungsstrategie sind des Weiteren die Auswahlkriterien der zu digitalisierenden Bestände festzuhalten. Eine Digitalisierung des gesamten analogen Bestandes ist für Archive oft kein kurzfristig, meist auch kein längerfristig zu erreichendes Ziel. Für kleinere Archive mit Beständen nur einer Person ist dies eher möglich; für nationale Einrichtungen wie das DLA oder SLA kaum. Obwohl dies durchaus als Fernziel formuliert werden kann, ist auch dann eine Priorisierungsleitlinie vonnöten. Eine Digitalisierungsstrategie muss diesbezüglich festlegen, aufgrund welcher Kriterien eine Archivalie oder ganze Bestände überhaupt und auf welche Weise digitalisiert werden soll(en). Eine ganze Reihe von Fragen geht dabei den entsprechenden Entscheidungen voraus, ${ }^{14}$ und die Systematisierung der Entscheidungsschritte ist für eine Digitalisierungsstrategie geboten, wie Becker und Oertel beschreiben. ${ }^{15}$

Auch die Ausführung der Digitalisierung (Workflow, Qualitätskontrolle und Outsourcing) muss in der Digitalisierungsstrategie festgehalten werden, ebenso wie die Festlegung der (Digitalisierungs-)Standards. Bei jeder Art der Digitalisierung von Archivgut ist vordringlich, "Zugänglichkeit, Benutzbarkeit und Authentizität digitaler Dokumente in der Gegenwart und insbesondere auch in der Zukunft über wechselnde technische Umgebungen sicherzustellen, sodass ihre ,significant properties',

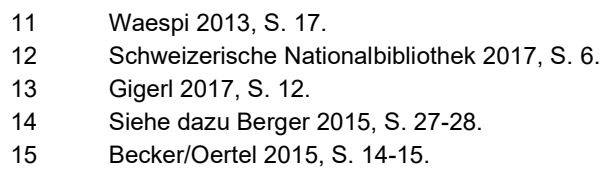


also ihre für die Authentizität konstitutiven, signifikanten Objekteigenschaften erhalten bleiben. " ${ }^{16}$ Für die archivische Erschliessung wurden internationale Standards entwickelt, die insbesondere im elektronischen Bereich einen internationalen Austausch ermöglichen. Um im Zuge der Vernetzung mit anderen Archiven auf Archivportalen den Zugang zu den eigenen Beständen zu gewährleisten, ist der Einsatz von Normdaten zentral. Als spartenübergreifendes Referenzsystem im deutschsprachigen Raum hat sich die Gemeinsame Normdatei GND etabliert. ${ }^{17}$ Solche normreferenzierten Daten können in das Semantic Web integriert werden und eröffnen ganz neue Möglichkeiten der Vernetzung von Inhalten, da sie eine multidimensionale Verzeichnung ermöglichen.

Literaturarchive richten sich bei der Erschliessung nach den 'Regeln zur Erschliessung von Nachlässen und Autographen' (RNA), ${ }^{18}$ die kürzlich durch die Arbeitsgruppe RNA/RDA ${ }^{19}$ im Rahmen des Standardisierungsausschusses ${ }^{20}$ überarbeitet wurden, was in den Richtlinien und Regeln für die 'Ressourcenerschliessung mit Normdaten in Archiven und Bibliotheken' (RNAB) resultierte. ${ }^{21}$ Die Experts Group on Archival Description (EGAD) des Internationalen Archivrats entwickelt zudem gerade einen neuen Standard für die archivische Erschliessung, 'Records in Contexts' (RiC), der in RNAB einbezogen und als neuer Standard bereits genannt wird. ${ }^{22}$ Ohne in diesem Rahmen auf die technischen Einzelheiten dieses neuen Standards eingehen zu können, ${ }^{23}$ soll festgehalten werden, dass er ganz neue Wege für die Benutzenden, aber auch für die Archive selber eröffnen wird: « Eine Verzeichnung von Archivgut nach RiC » eröffnet « die Möglichkeit, die entsprechenden Metadaten mit anderen zu verknüpfen - nicht allein über die Grenzen einer Institution, wir bei den bestehenden Archivportalen, sondern im Sinne von Linked Open Data auch über die Grenzen der Archivwelt hinaus. ${ }^{24}$

Kramski/Bülow 2017, S. 141.

Deutsche Nationalbibliothek 2019.

Für die RNA ist wiederum ISAD(G) richtungweisend, ein internationaler Anwendungsstandard zur Verzeichnung von Archivgut von Personen, Familien und Körperschaften, der im Jahr 2000 vom Internationalen Archivrat verabschiedet wurde.

Diese setzt sich redaktionell aus Vertretern des Literaturarchivs der Österreichischen Nationalbibliothek, der Handschriftenabteilung der Staatsbibliothek zu Berlin-Preussischer Kulturbesitz und der Schweizerischen Nationalbibliothek, die durch das SLA vertreten wird, zusammen. Die Moderation hat die Arbeitsstelle für Standardisierung der Deutschen Nationalbibliothek inne, und als Fachexperte ist u.a. auch das DLA vertreten.

Der Standardisierungsausschuss mit Vertretern aller grossen Bibliotheken der deutschsprachigen Länder hat zum Ziel, sicherzustellen, dass einheitliche Standards für die Erschliessung, Formate und Schnittstellen in Bibliotheken eingesetzt werden.

Österreichische Nationalbibliothek et al. 2019.

Österreichische Nationalbibliothek et al. 2019, S. 1.

Einen sehr guten Überblick bietet die Übersetzung eines Auszuges aus einer Präsentation von RiC anlässlich eines Kongresses in Seoul 2016, die die Arbeitsgruppe Normen und Standards des Vereins Schweizerischer Archivarinnen und Archivare erstellt hat (Verein Schweizerischer Archivarinnen und Archivare 2018).

Messner 2017. 
Der Einbezug des Umgangs mit den Linked (Open) Data ${ }^{25}$ in eine Digitalisierungsstrategie ist heutzutage unumgänglich: « Linked Open Data und Big Data werden ein integraler Bestandteil der zukünftigen Webinfrastruktur sein, in der grosse Datenmengen verfügbar und vernetzt sind. Diese Entwicklung wird auch Auswirkungen auf Archive, Bibliotheken und andere Informationseinrichtungen haben, selbst wenn diese bisher erst in Ansätzen erkennbar sind und das Potenzial von Linked Open Data und Big Data hier noch längst nicht ausgeschöpft ist. ${ }^{26}$ Für die Erschliessungsarbeit hat diese Entwicklung weitreichende Konsequenzen, und die " traditionellen Funktionen der Erschliessung, bzw. Beschreibung von Objekten mit Metadaten [erfahren] in der digitalen Welt eine Erweiterung und Neuausrichtung. " 27

Aus Nutzersicht ist in der Tat der früher « gewohnte Umfang der Erschließung den Zwecken des Findens, Identifizierens, Auswählens und Zugangs nicht angemessen. Er ist einerseits zu gering, wenn zum Beispiel nicht alle Autoren oder Interpreten eines Werks genannt werden, wenn die unselbstständigen Werke unterhalb des Zeitschriften-, Handbuch- oder Musik-CD-Titels nicht genannt werden oder wenn die Verlage nicht normiert verzeichnet werden, so dass für eine Rechte-Ermittlung nur mit viel Mühe und Fachwissen alle Titel eines Verlages in einem elektronischen Katalog abgefragt werden können. Es wird andererseits zuviel Aufwand betrieben, wenn rein deskriptiv in komplexen Kategorienschemata erfasst wird, was nicht zu einer Suche im elektronischen Katalog herangezogen werden kann - oder typischerweise wird. “28 Auf dieses ,Zuviel' an Aufwand rekurriert auch Bütikofer, wenn er festhält, dass es sich lohnen dürfte, " das manuelle Erschliessen auf einen harten Kern an standardisierten Metadaten zu beschränken » und die dabei « [f]rei werdende[n] Ressourcen [...] für die Digitalisierung » zu nutzen, " um oft überhaupt erst das Potential für die automatische Analyse und die vernetzte Nutzung zu schaffen, welche im Blick auf die Zukunft wichtiger sind, als eine detaillierte Beschreibung. " Dadurch, dass " Metadaten und Informationsobjekte [...] nicht in den eigenen Systemen vergraben werden, sondern im Sinne der Open Data-Bewegung anderen Institutionen für ihre Erschliessungsarbeit und unseren Nutzer/innen für ihre Auswertungen zur Verfügung gestellt werden », könnten schliesslich ganze « Informationslandkarten » entstehen. ${ }^{29}$

«'Linked Data' bezeichnet wohlstrukturierte, hoch konnektive und syntaktisch interoperable Datensets, die über mehrere Repositorien innerhalb einer Organisation oder organisationsübergreifend verteilt sind. [...] Dies gibt auch Archiven und Bibliotheken neue Möglichkeiten, ihre Bestände im Kontext des World Wide Webs zu erschliessen und zu katalogisieren.» Schlichte/Holländer 2013, S. 3. Schlichte/Holländer 2016, S. 3. Big Data sind in der vorliegenden Untersuchung nicht relevant, da es dabei um jegliche Arten von Daten geht, ob mit oder ohne Metadaten, die « allerorts durch die Vernetzung der Endgeräte (vom Grossrechner bis zum Smartphone und den darauf befindlichen Apps) ins Web eingespeist werden. " (Schneider 2013, S. 4) Dahinter stehen mehrheitlich wirtschaftliche Interessen. Linked Open Data dagegen macht « die gemeinnützige Publikation sowie eine Verknüpfung zur Conditio sine qua non » (Schneider 2013, S. 5). Bütikofer 2014, S. 7. 
Dies ist durch die bei der Erschliessung als Linked Data aufbereiteten Metadaten und digitalen Objekte bei deren Bereitstellung im Web möglich. RiC ist dabei der Versuch, " die Grundsätze archivischer Erschliessung unter grundlegend veränderten technischen Bedingungen neu zu formulieren. In anderen Worten: Mit RiC sollen die fundamentalen archivischen Prinzipien - das Provenienzprinzip und das Prinzip der Bewahrung und Verdeutlichung der ursprünglichen Zusammenhänge von Überlieferungsgut - ihren Platz erhalten in der Welt der verknüpfbaren Metadaten. " RiC bietet die Möglichkeit, " neu auch horizontale und nicht zuletzt plurale Beziehungen zwischen Entitäten » darzustellen; es bleibt dem archivischen Provenienzprinzip verpflichtet, doch mit « der Möglichkeit einer solchen sowohl-als-auch-Zuordnung erweitern sich die Gestaltungsmöglichkeiten bei der Verzeichnung von Archivbeständen enorm », was « vor allem Beständen mit komplexer Überlieferungsgeschichte zugute » kommt. ${ }^{30}$ Diese Komplexität der Überlieferungsgeschichte ist beispielsweise bei Nachlässen von AutorInnen oft gegeben, so dass diese technischen Entwicklungen einen grossen Nutzen gerade für Literaturarchive versprechen.

Schnittstellen stellen dabei sicher, dass Metadaten aus Bibliotheks- und Archivsystemen übernommen werden können: so sind Inhalte eines Systems auch in einem anderen System nachgewiesen und recherchierbar: « E-rara, e-manuscripta, Eperiodica und e-codices bieten zum Beispiel eine OAI-PMH-Schnittstelle zum Bezug der Metadaten und Links zu den Digitalisaten an. " ${ }^{31}$ Wenn die Nutzungsbedingungen durch Urheber- oder Persönlichkeitsrechte eingeschränkt werden müssen, kann eine nicht-offene Schnittstelle eingerichtet werden. Trotzdem können auch in einem solchen Fall die Daten als Linked Open Data aufbereitet werden, wie es beim Projekt letempsarchives.ch der EPFL gemacht wurde. ${ }^{32}$

Eine Digitalisierungsstrategie muss neben den Richtlinien zur (Retro-)Digitalisierung auch Richtlinien zu einer Open Access-Strategie enthalten und Aspekte des Umgangs mit dem Linked Open Data einbeziehen.

Ein weiterer Aspekt der Digitalisierungsstrategie ist die Festlegung der Bereitstellung von Daten. Die Nutzerinteressen und das Ziel, diese zu erfüllen, sind für Archive zwei der wichtigsten Gründe, überhaupt zu digitalisieren. Diese Nutzerinteressen geben generell auch vor, dass Material schnell und einfach gefunden werden muss und möglichst niederschwellig zur Verfügung stehen soll. Die Bereitstellung von Online-Findmitteln ist für die meisten Archive seit den 1990er Jahren üblich bzw. die Notwendigkeit, solche Findmittel zur Verfügung zu stellen, steht ausser Frage, und die Nutzenden sehen dies schon länger als eine Selbstverständlichkeit an. Inzwischen wird jedoch darüber hinaus auch erwartet, die Objekte digitalisiert online ansehen zu 
können. Eine adäquate Bereitstellung von digitalisiertem Archivgut ist zentral, damit dieses überhaupt genutzt wird; adäquat heisst in dem Fall benutzerorientiert: « Es wurde konstatiert, dass ein grosser Teil der digitalisierten Bestände in erster Linie nicht genutzt » wird, jedoch nicht aus mangelndem Interesse, sondern " weil die Aufbereitung und Präsentation nicht den Nutzerbedürfnissen » entspricht. ${ }^{33}$ Dass Archivgut, das für weitreichende Nutzungszwecke benutzt wird, nicht nur auf einem hauseigenen Kanal präsentiert werden sollte, sondern sinnvollerweise auch auf übergreifenden Archiv- oder Bibliotheksportalen, ist naheliegend. Kooperationen mit anderen Portalen und Plattformen sind sowohl in benutzungstechnischer als auch in finanzieller Hinsicht sinnvoll. ${ }^{34}$ Wo es sich bei den Plattformen um Portale handelt, auf denen Metadaten gesammelt und zur Verfügung gestellt werden, die konkreten Digitalisate jedoch weiterhin bei den jeweiligen Institutionen bleiben und darauf einfach verwiesen wird, " können hier auch spezifischere oder strengere Nutzungs- und Nachnutzungsregelungen Anwendung finden, während die Metadaten [...] für jeglichen Zweck frei nachnutzbar sind. ${ }^{35}$

Ein weiterer Aspekt der Digitalisierungsstrategie ist die Wartung der Bestände und die Digitale Langzeitarchivierung. Was Locher 2014 als « [t]echnische Hürden bei der Langzeiterhaltung digitaler Nachlässe » konstatiert hat, gilt heute noch: " Im Gegensatz zur Archivierung von Informationen auf Papier kann im digitalen Bereich nicht auf eine mehrere hundert Jahre alte Erfahrung zurückgegriffen werden. Die Archivinstitutionen lernen laufend dazu, tauschen sich aus und passen ihre Best Practices immer wieder den neuen Erkenntnissen an. ${ }^{36}$ Die von Locher beschriebenen grundlegenden Massnahmen für die Archivierung von digitalen Daten sind heute Standard: Kopieren der Daten von ihrem « ursprünglichen Datenträger in eine standardisierte Umgebung » zwecks einfacherer Pflege, wobei eine standardisierte technische Dokumentation wichtige Informationen speichern soll, wie beispielsweise die « Checksummer, mit Hilfe derer später überprüft werden kann, dass keine willkürliche Datenveränderung stattgefunden hat »; Ablage mehrfacher Kopien der Daten auf verschiedenen Speichersystemen und periodische Umkopierung der Daten auf neue Speichersysteme; sowie Migration und Emulation. ${ }^{37}$ Dennoch ist das ,Problemfeld Langzeitarchivierung' noch weit davon entfernt, gelöst zu sein. Da die Vorstellung von ,Langzeit‘ im traditionellen analogen Archivumfeld im Prinzip ,ewig' bedeutet, ist diese im digitalen Zeitalter insofern nicht einzulösen, als technische Entwicklungen unmöglich vorausgesehen werden können. Iordanidis spricht denn auch davon, dass « [p]raktisch [...] unter dem Begriff 'Langzeit' derzeit ein technisch überschaubarer Zeitraum von

33 Schubert 2018, S. 224

34 Mumenthaler/Schuldt/Wenk 2017, S. 6.

35 Arnold 2015, S. 24.

$36 \quad$ Locher 2014, S. 12.

$37 \quad$ Locher 2014, S. 11-12. 
5-10 Jahren verstanden » wird, " auch wenn das prinzipielle Ziel der dLZA [digitalen Langzeitarchivierung] die dauerhafte Nutzbarkeit von digitalen Ressourcen verfolgt. " ${ }^{38}$ Der Kommentar zur Norm DIN 31644 'Information und Dokumentation Kriterien für vertrauenswürdige digitale Langzeitarchive, ${ }^{39}$ definiert « den Begriff ,Langzeit‘ nicht als feste Zeitspanne (5, 10, 50 oder noch mehr Jahre), sondern als Ziel, die archivierten Objekte und Informationen über Veränderungen der Hard- und Software hinweg zu bewahren und zu nutzen. " ${ }^{40}$

\section{Herausforderungen und Chancen}

Die grössten Herausforderungen, aber auch Chancen für Privatarchive liegen ganz offensichtlich im Bereich des Semantic Web und der daraus resultierenden weltweiten Verknüpfung von Daten und digitalisierten Objekten des eigenen Archivs. Vom " grossen Potential der retrospektiven Digitalisierung »" können gerade Privatarchive mit Nachlassbeständen enorm profitieren: der Aspekt der Linked Open Data bietet beispielsweise die Möglichkeit, Dokumente, die zu einem Bestandsbilder gehören, aber nicht im eigenen Archiv lagern, mit dem eigenen Archivgut zu verknüpfen. So « könnten auf der ganzen Welt verstreute Archivbestände oder Nachlassbibliotheken anhand von Vorbesitzer- oder Aktenbildnervermerken virtuell zusammengeführt und an einem Ort digital konsultierbar gemacht werden. " ${ }^{42}$

Die Vorteile einer breiten Vernetzung liegen auf der Hand: « Größere Sichtbarkeit, vor allem für noch unerschlossene Nutzergruppen, und daraus folgende Nutzergewinnung » sowie « die Chance, Informationen über das Archiv selbst, über seine Bestände, das Archivgut und digitale Inhalte einer breiten Öffentlichkeit zu präsentieren. ${ }^{43}$ Die Digitalisierung eines Bestandes hat direkte Auswirkungen auf die Nachfrage, wie « das Beispiel des Nachlasses von Annemarie Schwarzenbach [zeigt]. Dieser ist der am zweithäufigsten nachgefragte Bestand des SLAs [...], was laut Gigerl mit der digitalisierten Fotosammlung in Zusammenhang steht. " ${ }^{44}$ Dies hat wiederum Auswirkungen auf den Bekanntheitsgrad eines Archivs und seiner Bestände selber, der sich « weiter durch regionale, institutionen-, länder-, sparten- oder themenübergreifende Recherchemöglichkeiten der jeweiligen Portale [steigert]. Erschließungsinformationen einer Institution werden in neue und ergänzende Zusammenhänge

$38 \quad$ Iordanidis 2014, S. 144.

39 Die deutsche Norm 31644 umfasst die gleichen Grundprinzipien und vergleichbare Anforderungen wie der internationale Standard ISO 16363, aufgrund dessen geprüft werden kann, ob ein Archivsystem vertrauenswürdig ist.

$40 \quad$ Keitel/Schoger 2013, S.3.

41 Mattmann 2016, S. 5.

$42 \quad$ Mattmann 2016, S. 4-5.

43 Arnold/Waidmann 2013, S. 432.

44 Siegrist 2017, S. 19. 
gebracht, welche wiederum neue Nutzergruppen zu erschließen helfen oder bestehenden Nutzergruppen neue Ansätze zu weiteren Recherchen bieten. » ${ }^{45}$ Dies führt ausserdem auch dazu, " dass konkrete Bestände der Kulturerbeeinrichtungen auch dann gefunden werden, wenn der Nutzer gar nicht bei der jeweiligen Institution sucht, in deren Obhut sie sich befinden und die für die Digitalisierung verantwortlich war, sondern durch einen Querverweis bei der Recherche in einem gänzlich anderen Zusammenhang und gegebenenfalls bei Beständen einer anderen Institution. » ${ }^{46}$

Für Archive mit privaten Nachlässen eröffnet sich durch die Verlinkung einzelner digitaler Bestände und Dokumente zudem die Möglichkeit, sowohl Pertinenzals auch Provenienz-Zusammenhänge sichtbar zu machen. In Studien zu Bedürfnissen von Nutzenden hat sich gezeigt, " dass NutzerInnen verwirrt waren, wenn Resultate nach Provenienz aufgelistet waren ", denn viele von ihnen, " vor allem Nicht-HistorikerInnen ", sind offenbar « nicht gewillt oder geschult [...], einen Bestand oder Dokumente über die Provenienz zu (durch-)suchen. » ${ }^{47}$ Die Empfehlung, Suchresultate deshalb nicht nach Provenienz zu ordnen, widerspricht jedoch dem archivischen Prinzip der Darstellung von Ursprungszusammenhängen von Überlieferungsgut. Wie gezeigt wurde, versprechen die neusten Entwicklungen im Bereich der RiC hier eine Lösung, die es durch die auf Archivseite mögliche Sowohl-als-auch-Zuordnung den Nutzenden erlauben wird, je nach Nutzerinteresse verschiedene Darstellungen desselben Bestandes abzurufen.

Digitalisierungsstrategien lassen sich bei der Formulierung ihrer Ziele sehr oft von Restriktionen leiten. Obgleich beispielsweise sowohl das DLA als auch das SLA die Wichtigkeit der digitalen Erschliessung von Metadaten und ihrer Aufbereitung nach Standards, die eine Verknüpfung der Daten im Semantic Web erlauben, erkannt haben und hier Vorreiterrollen einnehmen, ${ }^{48}$ sind beide Institutionen bisher äusserst zurückhaltend, was die (Retro-)Digitalisierung des Bestandes selber betrifft. Diese Diskrepanz ist dadurch zu erklären, dass von der Standardisierung der Erschliessung der Metadaten in den beiden Institutionen auch die institutionell verbundenen Bibliotheken profitieren, die in dem Bereich überdies bereits weiter fortgeschritten sind, da gerade der neue Standard RDA zunächst für Bibliotheken konzipiert wurde. Die Archive sehen sich nun in der Position der darauf reagierenden Partei, die dafür sorgen muss, dass der Standard möglichst optimal auch für Archive anwendbar wird. Dabei wird offenbar vergessen, dass die Digitalisierung der eigenen Bestände indes ein immens wichtiger Bestandteil im Projekt des Semantic Web darstellt, der den Archiven eine führende Position in diesem Projekt bieten könnte. 
Selbstverständlich haben die Institutionen immer mit Restriktionen zu kämpfen, die das Erreichen ehrgeiziger Ziele zu verunmöglichen scheinen. Realistische Einschätzungen bezüglich Mangel an Ressourcen und juristischen Beschränkungen können das allzu optimistische Formulieren von Visionen hemmen. Doch die Untersuchung hat gezeigt, dass die Gründe, die zu solcher Zurückhaltung führen, in vielen Fällen neu überdacht werden sollten. Vor allem die juristischen Implikationen der Bereitstellung von Material, das urheber- oder persönlichkeitsrechtlich geschützt ist, führen zu einer äusserst zurückhaltenden Handhabung der Digitalisierung von Archivgut. Dabei ist deutlich, dass auch geschütztes Material durchaus digitalisiert werden darf.

Es wäre in dieser Hinsicht zu überlegen, ob ein Literaturarchiv, das in eine grössere Institution eingebunden ist, hier nicht in einer für sich selber formulierten Digitalisierungsstrategie eine abweichende Haltung einnehmen und sich vornehmen könnte, innerhalb eines Projekts durchaus auch geschützte Werke zu digitalisieren. Dies wäre damit zu begründen, dass auf diese Weise sukzessive auch Altbestände nicht nur digitalisiert, sondern auch digital erschlossen werden. Laut den Richtlinien der NB werden diese nämlich nur dann digital erschlossen, wenn Nachfrage besteht. Unter Berücksichtigung der in der Untersuchung aufgezeigten Mechanismen, dass nur digital erschlossene Bestände im Semantic Web verarbeitet und verknüpft werden können und sich die Nachfrage gerade dadurch reguliert (,nur was digital vorhanden ist, wird erforscht'), ist ein solches Vorgehen nicht sinnvoll. Vielmehr sollte es aus den soeben geschilderten Gründen das Ziel sein, alle Bestände zumindest digital zu erschliessen. Natürlich sind einem solchen Vorhaben ressourcenbedingte Schranken gesetzt und muss auf jeden Fall priorisiert werden. Doch unter der Perspektive der enormen Chance, die ein solches Unterfangen bietet, können die Auswahlkriterien neu gewichtet werden. So ist es durchaus denkbar, bei der manuellen Erschliessung weniger in die Tiefe zu gehen und die frei so werdenden Ressourcen bei der Digitalisierung einzusetzen, wie Bütikofer vorschlägt. ${ }^{49}$

Da sich die Arbeitsprozesse im Archiv gegenwärtig in einem tiefgreifenden Wandel befinden, sind solche Neugewichtungen der Priorisierungen bei der Verteilung von Ressourcen durchaus sinnvoll. Die von Glauert noch 2013 formulierte Forderung, dass « das große öffentliche, wissenschaftliche und politische Interesse an einer Online-Bereitstellung von Archivgut am Ende natürlich nicht zu einer Umschichtung öffentlicher Gelder oder personeller Ressourcen von der Erschließung oder Originalerhaltung in die Digitalisierung führen ${ }^{50}$ dürfe, kann heutzutage nicht mehr auf diese Weise vorgebracht werden. Abgesehen davon, dass Digitalisierung von analogem Archivgut sowieso der Originalerhaltung zu Gute kommt, ist unter der Perspektive der Linked (Open) Data auch die Erschliessung selber nicht mehr als 
eigenständiger Prozess neben dem Prozess der Digitalisierung zu sehen. Erschliessung und Aufbereitung von Metadaten sind insofern ein Teil der Digitalisierung geworden, als die digitalen Erschliessungsinformationen den Schlüssel bilden zur Verbreitung und Verknüpfung von Daten und Digitalisaten. Eine Neuorientierung bei der Priorisierung der Ressourcenverteilung in den einzelnen archivischen Prozessen ist deshalb plausibel.

Abschliessend ist festzuhalten, dass sich langfristig, wie Mattmann es formuliert, die « Inangriffnahme dieses (Mammut-)Projekts [Digitalisierung] in jeder Hinsicht lohnen » wird. ${ }^{51}$

\section{Literaturverzeichnis}

Arnold, Kerstin: Europas Kulturgut online - die Europeana. Europas Kulturgut online - die Europeana. In: Archivar, H. 1 (2015), S. 24-27.

Arnold, Kerstin; Susanne Waidmann: Vernetzte Präsentation. Erfahrungen mt Portalen. In: Archivar, H. 4 (2013), S. 431-438.

Becker, Irmgard Christa; Oertel, Stephanie: Priorisierung ist von den Zielen der Digitalisierung abhängig! Ergebnisse des Workshops am 6. Mai 2013 und Vorstellung der Entscheidungsmatrix. In: Dies. (Hg.): Digitalisierung im Archiv Neue Wege der Bereitstellung des Archivguts. Beiträge zum 18 . Archivwissenschaftlichen Kolloquium der Archivschule Marburg. Marburg 2015, S. 13-22.

Berger, Andreas: Digitalisierung eines ganzen Archivs? Strategien zur Auswahl, Priorisierung und Durchführung von Massendigitalisierungen. In: Becker, Irmgard Christa; Oertel, Stephanie (Hg.): Digitalisierung im Archiv - Neue Wege der Bereitstellung des Achivguts. Beiträge zum 18. Archivwissenschaftlichen Kolloquium der Archivschule Marburg. Marburg 2015, S. 23-32.

Bountouri, Lina: Archives in the Digital Age: Standards, Policies and Tools. Cambridge 2017.

Bütikofer, Niklaus: Erschliessungstheorie und AV-Dokumente. In: arbido, H. 2 (2014), S. 4-7.

Deutsche Nationalbibliothek: Gemeinsame Normdatei (GND). https://www.dnb.de/DE/Professionell/Standardisierung/GND/gnd_node.html

Diesterhöft, Martin: Persönlichkeits- und datenschutzrechtliche Probleme der digitalen Zugangseröffnung zu analogen Inhalten durch Bibliotheken und Archive. In: Hinte, Oliver; Steinhauer, Eric (Hg.): Die Digitale Bibliothek und ihr Recht - ein Stiefkind der Informationsgesellschaft? Kulturwissenschaftliche Aspekte, technische 
Hintergründe und rechtliche Herausforderungen des digitalen kulturellen Speichergedächtnisses. Münster 2014, S. 51-84.

Gigerl, Margit: Der digitale Rilke. In: Passim 20 (2017), S. 12.

Gillner, Bastian: Archive im digitalen Nutzerkontakt. Virtuelle Lesesäle, soziale Medien und mentale Veränderungszwänge. In: Archivar, H. 4 (November 2013). S. 406-415.

Glauert, Mario: Dimensionen der Digitalisierung. Kosten, Kapazitäten und Konsequenzen. In: Claudia Kauertz (Red.): Digital und Analog. Die beiden Archivwelten. 46. Rheinischer Archivtag 21.-22. Juni 2012 in Ratingen. Beiträge. Bonn 2013, S. 48-59.

Heckmann, Jörn: Kapitel 5: Retrodigitalisierung. In: Gerald Spindler (Hg.): Rechtliche Rahmenbedingungen von Open Access-Publikationen. Göttingen 2006, S. 123-148.

Iordanidis, Martin: Digitale Langzeitarchivierung - Risikoebenen und Lösungsstrategien im nachhaltigen Umgang mit digitalen Ressourcen. In: Hinte, Oliver; Steinhauer, Eric W.: Die Digitale Bibliothek und ihr Recht - ein Stiefkind der Informationsgesellschaft? Kulturwissenschaftliche Aspekte, technische Hintergründe und rechtliche Herausforderungen des digitalen kulturellen Speichergedächtnisses. Münster 2014, S. 141-160.

Källman, Rolf: Digisam - towards a Coordinated Digital Cultural Heritage in Sweden. In: Becker, Irmgard Christa; Oertel, Stephanie (Hg.): Digitalisierung im Archiv - Neue Wege der Bereitstellung des Archivguts. Beiträge zum 18. Archivwissenschaftlichen Kolloquium der Archivschule Marburg. Marburg 2015, S. 33-48.

Keitel, Christian; Schoger, Astrid (Hg.): Vertrauenswürdige digitale Langzeitarchivierung nach DIN 31644. Berlin, Wien, Zürich 2013.

Klimpel, Paul; Rack, Fabian; Weitzmann, John H.: Handreichung. Neue rechtliche Rahmenbedingungen für Digitalisierungsprojekte von Gedächtnisinstitutionen. 4., gänzlich neu bearbeitete Auflage. Berlin 2017.

Kramski, Heinz Werner; Bülow, Ulrich von: 'Es füllt sich der Speicher mit köstlicher Habe' - Erfahrungen mit digitalen Archivmaterialien im Deutschen Literaturarchiv Marbach. In: Robertson-von Trotha, Caroline Y.; Hauser, Robert (Hg.): Neues Erbe. Aspekte, Perspektiven und Konsequenzen der digitalen Überlieferung. OpenEdition Books 2017, S. 141-162.

Kwasnitza, Stefan: Der normierte Sucheinstieg - GND und RDF. In: arbido, H. 4 (2014), S. 46-47.

Locher, Hansueli: Technische Hürden bei der Langzeiterhaltung digitaler Nachlässe. In: Passim 14 (2014), S. 11-12. 
Mattmann, Beat: Die digitale Zugänglichkeit von Archivalien: Stand der Dinge aus Praxissicht. In: Informationspraxis Bd. 2, Nr. 1 (2016).

Messner, Philipp: Records in Contexts - vom Baum zum Netz. In: arbido, H. 3 (2017).

Mumenthaler, Rudolf; Schuldt, Karsten; Wenk, Bruno: Bericht: Analyse der Informationsarchitektur, Schnittstellen und Organisation der Online-Plattformen (AISOOP). Eine Studie der HTW Chur, gefördert im Rahmen des Programms "Wissenschaftliche Information" (SUK 2013-2016 P-2) von swissuniversities. Chur 2017.

Niggemann, Elisabeth: Die Notwendigkeit des revolutionären Katalogisierens. In: Menne-Haritz, Angelika; Hofmann, Rainer (Hg.): Archive im Kontext. Öffnen, Erhalten und Sichern von Archivgut in Zeiten des Umbruchs. Düsseldorf 2010, S. 241-251.

Österreichische Nationalbibliothek, Schweizerische Nationalbibliothek, Staatsbibliothek zu Berlin-Preussischer Kulturbesitz: Ressourcenerschliessung mit Normdaten in Archiven und Bibliotheken (RNAB) für Personen-, Familien-, Körperschaftsarchive und Sammlungen. Richtlinie und Regeln. Version 1.0 (Januar 2019). Frankfurt/M. 2019. https://d-nb.info/1186104252/34 (4.7.2019)

Pilger, Kathrin: Editorial. In: Archivar, H. 3 (2015), S. 213.

Schlichte, Annkristin; Holländer, Stephan: Editorial. Linked Open Data und Big Data. In: arbido, H. 4 (2013), S. 3.

Schmidgall, Karin: Die SRU Update Schnittstelle - Erschließung mit Zukunft. In: bit.online 18/4 (2015), S. 343-347.

Schneider, René: Dimensionen und Zusammenhänge grosser, verknüpfter, offener und wissenschaftlicher Daten. In: arbido, H. 4 (2013), S. 4-7.

Schubert, Irina: Nutzerbedürfnisse für retrodigitalisierte Primärquellen. Eine Metastudie und eine vergleichende Studie unter Gedächtnisinstitutionen. In: Informationswissenschaft: Theorie, Methode und Praxis (2018), S. 220-250.

Schweizerische Nationalbibliothek: Digitalisierungsleitline. Juli 2014 (Stand März 2017). https://www.nb.admin.ch/dam/snl/de/dokumente/epublikationen/publikationen/digitalisierungsleitlinienb.pdf.download.pdf/digitalisier ungsleitlinienb.pdf (4.7.2019)

Schweizerische Nationalbibliothek: Strategie 2012 bis 2019. Version 2014. https://www.nb.admin.ch/dam/snl/de/dokumente/nb_als_flag-einheit/studien_und_berichte/nb_strategie_2012-2019version2014.pdf.download.pdf/nb_strategie_2012-2019version2014.pdf (4.7.2019)

Siegrist, Franziska: Auswahlkriterien für die Digitalisierung archivalischer Quellen. Kriterienkatalog und seine praktische Anwendung auf ausgewählte Bestände 
des Schweizerischen Literaturarchivs. Unveröffentlichte CAS ALIS Abschlussarbeit 2017.

Söring, Sibylle: Technische und infrastrukturelle Lösungen für digitale Editionen: DARIAH-DE und TextGrid. In: Bibliothek - Forschung und Praxis 40:2 (2016), S. 207-212.

Verein Schweizer Archivarinnen und Archivare: Records in Contexts (RiC). Entwurf des archivischen Erschliessungsstandards. Übersetzung eines Auszuges der Präsentation anlässlich der Vorstellung des Standards in Seoul im September 2016. http://vsa-aas.ch/wp-content/uploads/2016/11/20161122_ICA-EGAD-Folien$\%$ C3\%9Cbersetzung-Deutsch.pdf (4.7.2019)

Waespi, Oliver: Aspekte des Urheberrechts im Literaturarchiv. In: arbido, H. 1 (2013), S. 17-19. 